\title{
A Unique Role for Endothelial Cell Kinesin Light Chain 1, Variant 1 in Leukocyte Transendothelial Migration
}

Bita F. Cyrus and William A. Muller

From the Department of Pathology, The Feinberg School of Medicine, Northwestern University, Chicago, Illinois

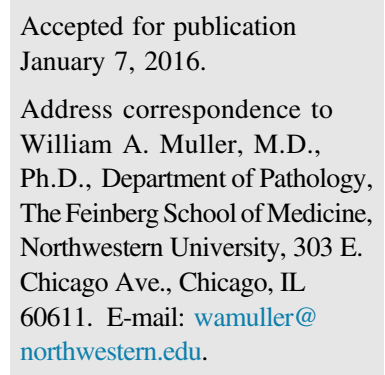

Address correspondence to William A. Muller, M.D., Ph.D., Department of Pathology, The Feinberg School of Medicine, Northwestern University, 303 E. Chicago Ave., Chicago, IL 60611. E-mail: wamuller@ northwestern.edu.

\begin{abstract}
A reservoir of parajunctional membrane in endothelial cells, the lateral border recycling compartment (LBRC), is critical for transendothelial migration (TEM). We have previously shown that targeted recycling of the LBRC to the site of TEM requires microtubules and a kinesin molecular motor. However, the identity of the kinesin and mechanism of cargo binding were not known. We show that microinjection of endothelial cells with a monoclonal antibody specific for kinesin-1 significantly blocked LBRC-targeted recycling and TEM. In complementary experiments, knocking down KIF5B, a ubiquitous kinesin-1 isoform, in endothelial cells significantly decreased targeted recycling of the LBRC and leukocyte TEM. Kinesin heavy chains move cargo along microtubules by one of many kinesin light chains (KLCs), which directly bind the cargo. Knocking down KLC 1 isoform variant 1 (KLC1C) significantly decreased LBRC-targeted recycling and TEM, whereas knocking down other isoforms of KLC1 had no effect. Re-expression of KLC1C resistant to the knockdown shRNA restored targeted recycling and TEM. Thus kinesin-1 and KLC1C are specifically required for targeted recycling and TEM. These data suggest that of the many potential combinations of the 45 kinesin family members and multiple associated light chains, KLC1C links the LBRC to kinesin-1 (KIF5B) during targeted recycling and TEM. Thus, KLC1C can potentially be used as a target for anti-inflammatory therapy. (Am J Pathol 2016, 186: 1375-1386; http://dx.doi.org/10.1016/j.ajpath.2016.01.011)
\end{abstract}

The inflammatory response is crucial for eliminating foreign microorganisms and healing wounds, but uncontrolled inflammation is responsible for most pathologic processes, including diseases such as atherosclerosis and rheumatoid arthritis. A critical step in the inflammatory response is diapedesis, or transendothelial migration (TEM). ${ }^{1-3}$ In this step, leukocytes move across the endothelium, into the damaged tissue. This occurs most often at endothelial cell borders (paracellular migration). To reach a site of inflammation in the lumen of epithelial-lined organs, leukocytes have to cross the epithelium in addition to the endothelium to get to the site of inflammation. ${ }^{4}$ Most of the benefits and most of the collateral damage that accumulate as a result of inflammation occur when leukocytes infiltrate the affected tissues. Thus, molecules that regulate TEM make logical targets for antiinflammatory therapy.

We have discovered a novel membrane compartment in endothelial cells that regulates TEM: the lateral border recycling compartment (LBRC). ${ }^{5,6}$ As the name implies, the LBRC consists of interconnected membrane vesicle-like structures that cycle between the parajunctional region and the junctional surface of the endothelium. The LBRC is distinct from typical recycling endosomes, vesiculo-vacuolar organelles, and caveolae. ${ }^{5,7}$ Moreover, because it is always connected in some places to the lateral border, it is never totally internalized and can be thought of as tortuous invaginations of the lateral border membrane. ${ }^{5,8,9}$ Thus, targeted recycling is a term used for convenience; membrane fusion is not necessarily involved.

The LBRC contains a subset of membrane proteins, such as platelet endothelial cell adhesion molecule 1 (PECAM-1), poliovirus receptor, ${ }^{10}$ CD99, and junctional adhesion

Supported by NIH grants F31HL114374 (B.F.C.), R01 HL046849 (W.A.M.), and R37 HL064774 (W.A.M.).

Disclosures: None declared. 
molecule-A, that are required for TEM. ${ }^{11}$ At rest, membrane moves constitutively between the LBRC and the endothelial cell borders with a half-time of approximately 10 minutes. ${ }^{5}$ In this constitutive process LBRC membrane recycles evenly along the endothelial borders. ${ }^{5,6}$ During TEM, however, membrane from the LBRC is directed to the site at the endothelial cell borders where the leukocyte is transmigrating. This targeted recycling facilitates TEM by delivering membrane surface area and specific adhesion/signaling molecules to the migrating leukocyte. Inhibiting targeted recycling of the LBRC blocks leukocyte TEM. ${ }^{5}$

We have shown that targeted recycling of the LBRC requires functional microtubules and kinesin molecular motors. ${ }^{6}$ Microinjection of endothelial cells with a polyclonal antibody against the conserved motor domain of Drosophila conventional kinesin inhibited targeted recycling and TEM to a similar magnitude as blocking PECAM or depolymerizing microtubules. ${ }^{6}$ Leukocytes were still able to adhere to the endothelial cells and move to the junctions, but they could not migrate between junctions, suggesting a specific role for kinesin function in the movement of the LBRC during targeted recycling and TEM. However, the polyclonal antibody we used (HD) is capable of blocking many different kinesins. ${ }^{12}$

Here, we show that of the hundreds of potential combinations of the 45 kinesin heavy chains (encoded by 45 $K I F$ genes) that comprise 15 kinesin families and multiple associated light chains, only kinesin-1 in combination with kinesin light chain 1 isoform variant 1 (KLC1C) has this effect. With the use of shRNA knockdown and function-blocking antibody microinjection studies we show that $K I F 5 B$, the ubiquitous version of mammalian kinesin-1 is the motor responsible for targeted LBRC recycling required for monocyte TEM. Kinesin1 -associated light chains tether cargo to kinesin- $1 .{ }^{13-15}$ In some cases, kinesin-1 heavy chains bind their cargo directly. ${ }^{16,17}$ Here, we show that only splice variant 1 of kinesin light chain 1 (KLC1) is required for mediating TEM. KLC1 variant 1 is thus a potential novel target for anti-inflammatory therapy, because selectively disrupting its function would block leukocyte transmigration.

\section{Materials and Methods}

\section{Cells and Plasmid Constructs}

Human umbilical vein endothelial cells (HUVECs) were isolated as described ${ }^{18}$ and used for all experiments. They were grown on collagen and fibronectin, in medium 199 (M199; which includes bicarbonate and HEPES) supplemented with $20 \%$ human serum and $5 \%$ penicillin/ streptomycin. ${ }^{19}$

The kinesin-1 (NM_004521) knockdown sequences were as follows: 5'-CTCAAGAGCAAGTGTATAAT-3' and $5^{\prime}$-AAAGATGTACTTGAAGGATAT- ${ }^{\prime}$. The kinesin heavy chain (KHC) rescue construct was made with silent point mutations in the targeted sequences and was cloned into the vector that contained an in-frame mCherry tag at the $3^{\prime}$ end to distinguish the rescue construct from endogenous kinesin-1. The KLC shRNAs were generated to target the $3^{\prime}$ noncoding region of the respective mRNAs. The sequences are as follows: KLC1 isoform variant 1 (NM_005552.4): $5^{\prime}$-TGTATTTGTGTCTTTCTAA-3'; KLC1 isoform variants 2 (NM_182923.3) and 3 (NM_001130107.1): 5'-GCATAGGACATGATACTAA-3' ${ }^{\prime}$ The KLC1C was rescued by KLC1 variant 1 cDNA (ViGene Biosciences, Inc., Rockville, MD).

\section{Antibodies}

SUK4 (anti-kinesin-1) hybridoma cell line and K2.4 (anti-kinesin-2) ascites were gifts from Dr. Vladimir Gelfand (Northwestern University, Evanston, IL). AntiKinesin 5B polyclonal antibody was purchased from Sigma-Aldrich (St. Louis, MO). Rabbit monoclonal antibody (mAb) against KLC1 was purchased from Abcam (Cambridge, UK). Rabbit pAb against KLC2 was purchased from Pierce Antibodies (Rockford, IL). Unlabeled and Alexa 568-conjugated goat anti-mouse $\mathrm{F}\left(\mathrm{ab}^{\prime}\right)_{2}$ antibodies from Jackson ImmunoResearch Laboratories (West Grove, PA) and Life Technologies (Carlsbad, CA) were used for targeted recycling experiments to label recycling PECAM. Hec1 (anti-vascular endothelial-cadherin), hec7 (anti-CD31), P1.1 [anti-CD31, a gift from Dr. Peter Newman (Blood Center of Wisconsin, Milwaukee, WI)], IB4 (anti-CD18), and hec2 (anti-CD99) were used as previously described. ${ }^{5,20}$ Anti- $\boldsymbol{\alpha}$-tubulin antibody was purchased from Accurate Chemical (Westbury, NY), and anti- $\beta$-actin antibody was purchased from Abcam.

\section{Transduction of HUVECs with Adenovirus}

293A cells were grown on $100-\mathrm{mm}$ dishes to $70 \%$ to $90 \%$ confluence and then transfected with adenoviral constructs with the use of Lipofectamine 2000 for delivery. Amplified virus that expressed KHC and KLC knockdown and rescue constructs was purified and used in transduction of HUVECs for knockdown and rescue experiments (ViraPower Adenoviral Expression System; Thermo Fisher Scientific, Waltham, MA).

\section{SDS-PAGE and Western Blot Analysis}

HUVECs were harvested for SDS-PAGE by washing with phosphate-buffered saline (PBS) and adding sample buffer. DNA was sheared by passing through a 27 -gauge needle 10 times, and samples were heated to $100^{\circ} \mathrm{C}$ for 5 minutes. Samples were separated on $8 \%$ polyacrylamide gels and transferred onto polyvinyldifluoride membranes before blocking with 5\% milk in PBS with $0.05 \%$ Tween and probing with primary antibodies. Blots were incubated with horseradish peroxidase-conjugated secondary antibodies in 5\% milk in PBS with $0.05 \%$ Tween, then washed, and detected with chemiluminescence and exposure to X-ray film. 


\section{Microinjection into HUVECs}

HUVECs were plated at 30,000 cells per Mattek dish in M199 supplemented with 20\% human serum, 5\% penicillin/ streptomycin, and bicarbonate for 2 days before microinjection. On the day of microinjection, HUVECs were activated with $20 \mathrm{ng} / \mathrm{mL}$ tumor necrosis factor- $\alpha$, and media was exchanged to M199 without bicarbonate and supplemented with $20 \%$ fetal bovine serum. A working concentration of $5 \mathrm{mg} / \mathrm{mL}$ SUK4 mouse mAb, preimmune mouse IgG isotype control, and $\mathrm{K} 2.4$ were mixed with $1 \mathrm{mg} / \mathrm{mL}$ Dextran-Alexa 488 (purchased from Thermo Fisher Scientific) for microinjection to tag injected cells. Microinjection was performed under an Olympus DSU microscope $(20 \times$ phase contrast lens), with the use of an Eppendorf FemtoJet Microinjector and Micromanipulator. Cells were kept at $37^{\circ} \mathrm{C}$ during microinjection. Microcapillaries were pulled with a P-97 Flaming/Brown Micropipette Puller (Sutter Instrument, Novato, CA). A field of approximately 100 contiguous HUVECs was microinjected with the SUK4 $\mathrm{mAb}$. After microinjection, HUVEC media were exchanged back to conditioned M199 with 20\% human serum, 5\% penicillin/streptomycin, and bicarbonate, and then placed in a $37^{\circ} \mathrm{C} \mathrm{CO}_{2}$ incubator to allow for HUVECs to recover for at least 1 hour before conducting targeted recycling assays.

\section{TEM Assay}

The TEM assay is a quantitative assay designed to assess, using large numbers of transmigration events $(>300$ per variable tested), the effect of various treatments such as knocking down kinesin-1, on the process of transmigration. As routinely performed in our laboratory, ${ }^{19,21}$ peripheral blood mononuclear cells (to assess monocyte transmigration) from healthy volunteers are purified on a Ficoll density gradient and then washed in Hanks' buffered saline solution plus $0.1 \%$ human serum albumin. After this wash, they are resuspended in M199 plus 0.1\% human serum albumin. Cells $\left(2 \times 10^{6}\right)$ were added to confluent tumor necrosis factor- $\alpha$-activated (10 ng/mL, $>4$ hours) HUVEC monolayers grown on hydrated collagen gels and incubated for 1 hour at $37^{\circ} \mathrm{C}$ in a $\mathrm{CO}_{2}$ incubator. ${ }^{22}$ This is more than enough time to allow maximum TEM of control cells (approximately 90\%).

To assess the role of microtubule motors in leukocyte TEM, control and experimentally treated HUVECs were compared side by side in this assay. The co-culture was washed with cold PBS and fixed overnight with 2.5\% glutaraldehyde in $0.1 \mathrm{~mol} / \mathrm{L}$ sodium cacodylate buffer, $\mathrm{pH}$ 7.4. TEM was quantitated by Nomarski optics, as previously described. ${ }^{21}$ To image, the cells plated on collagen gels in 96-well plates were transferred onto glass slides and visualized by a Zeiss Axiophot 2 microscope (Carl Zeiss Inc., Thornwood, NY), with a $60 \times$ oil immersion objective. Adherent monocytes are those in the same focal plane as the endothelial monolayer. Transmigrated monocytes are those in a focal plane below the monolayer. Monocytes were scored in each of several random fields for each monolayer until at least 100 were counted, with three replicate monolayers for each condition for a total of $>300$ cells per variable tested. To avoid unintentional bias, all cells in the field were counted. Data are expressed as the percentage of total leukocytes (attached plus transmigrated) that have transmigrated. Each experiment was repeated at least three times.

\section{Targeted Recycling Assay}

The targeted recycling assay determines whether the LBRC has been recruited to transmigrating leukocytes. Targeted recycling was performed as described previously. ${ }^{5}$ In short, HUVEC monolayers were incubated with an Fab fragment of P1.1 mAb for 1 hour at $37^{\circ} \mathrm{C}$ in a $\mathrm{CO}_{2}$ incubator. P1.1 binds to PECAM domain five and does not interfere with any known function of PECAM. ${ }^{23}$ HUVECs were then put on ice and washed with PBS to remove any free unbound $\mathrm{Fab}$, followed by an incubation with an excess of $\mathrm{F}\left(\mathrm{ab} \mathrm{b}_{2}\right.$ fragment of unlabeled goat anti-mouse $\operatorname{IgG}$ on ice for 1 hour. This allows saturable binding of all of the Fab that is present on the surface of the endothelial junction, but it prevents entry to the LBRC at this temperature. Again, free antibody was washed away and $2 \times 10^{6}$ peripheral blood mononuclear cells mixed with Alexa 568-conjugated $\mathrm{F}\left(\mathrm{ab}^{\prime}\right)_{2}$ fragment of the same goat anti-mouse antibody were added to the cells for 20 minutes on ice and then transferred to the $\mathrm{CO}_{2}$ incubator for 8 minutes to allow for synchronized transmigration to begin. Cells were then put on ice and washed with ice-cold PBS, followed by fixation in $2 \%$ paraformaldehyde for 10 minutes at room temperature.

Because the P1.1 mAb does not interfere with PECAM function, tracking it serves as a surrogate marker for the movement of the LBRC. In this procedure, only PECAM that was originally in the LBRC and recycled to the surface during the transmigration period will be labeled with the fluorescent secondary antibody, because it was protected from the unlabeled secondary antibody during the incubation on ice. The synchronized transmigration for a short time (8 minutes) catches leukocytes early in the act of transmigration before significant constitutive recycling and a corresponding increase in the staining of uninvolved junction occurs.

Confocal fluorescence microscopy was used to image the targeted recycling events. Leukocytes were identified with fluorescently conjugated mAb against CD18 (IB4). Diapedesis was determined by demonstration of leukocytes traversing the endothelial monolayer as seen on multiple sequential focal planes and with the use of an orthogonal projection. We assessed the number of monocytes in contact with the endothelial monolayer at cell junctions and those that were contacted by membrane from the LBRC. We measure the intensity of fluorescent goat anti-mouse mAb to quantify enrichment 
A
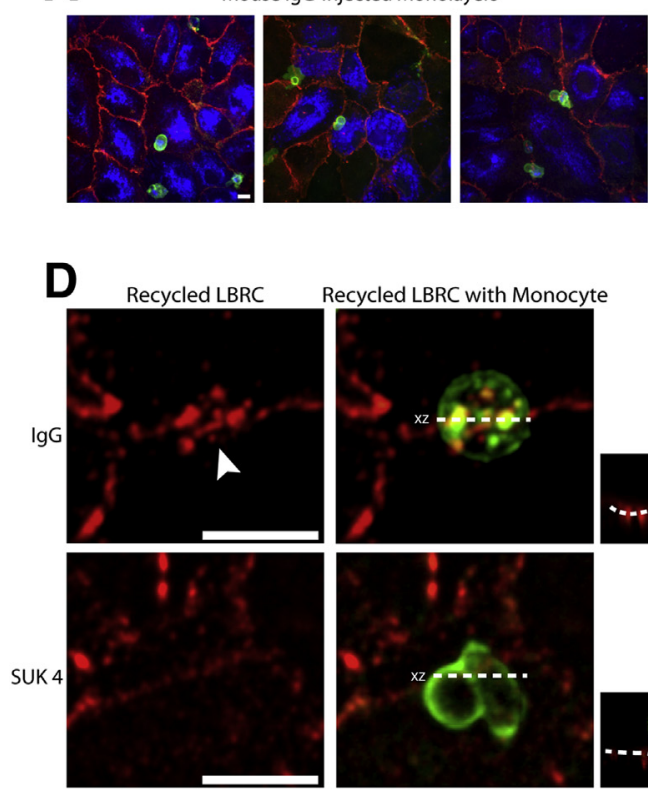

B

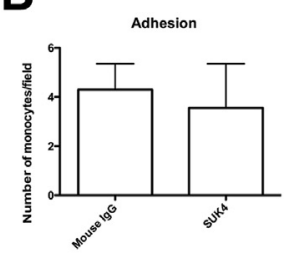

$\mathbf{E}$

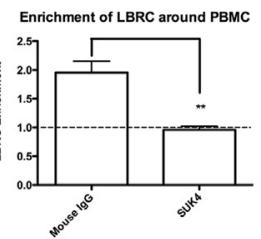

C

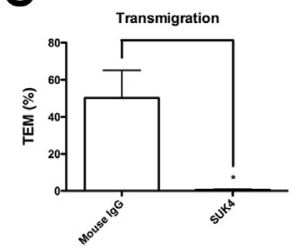

$\mathbf{F}$

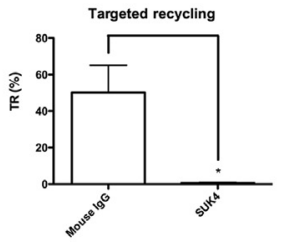

G

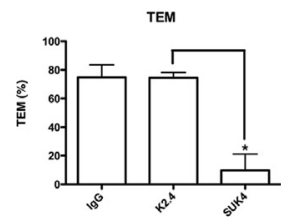

Figure 1 Blocking kinesin-1 function via microinjection of SUK4 in HUVECS inhibits TEM and TR. HUVECs were microinjected with SUK4 (kinesin-1 mAb) or isotype-matched mouse IgG control antibody. Antibodies were mixed with a fluorescent-conjugated dextran to label injected cells. Monocytes were allowed to settle on the monolayer and then transmigrate for 7.5 minutes. A: HUVEC monolayers (labeled by PECAM in red) remained intact after microinjection (microinjected cells labeled blue) and monocyte (labeled green) TEM. B and C: Confocal stacks were imaged, and the numbers of PBMCs that have attached and migrated to EC junctions were counted (B), and TEM was quantified (C). D: High-power images of confocal stacks to show differences in TEM and TR in SUK4- versus IgG control-microinjected cells. Constitutive recycling occurs evenly but spottily along the junctions; however, TR enhances LBRC fluorescence at sites of TEM. Orthogonal projections ( $\mathrm{xz}$ ) are depicted as the smaller images to the right of their corresponding images. The monocyte shown for the IgG control-microinjected cells is just starting TEM, as seen in the orthogonal projection. Arrowhead indicates site of leukocyte TEM. Dotted lines in the orthogonal projection indicate abluminal surface of endothelial cells. E: LBRC enrichment was measured around leukocytes at endothelial junctions. F: TR significantly diminishes after microinjection of SUK4 mAb against kinesin-1. G: TEM is significantly lower in SUK4-injected cells compared with cells injected with K2.4 (anti-kinesin-2). Data are expressed as means \pm SEM. $n=3$ experiments with two monolayers per condition for each experiment and at least 100 monocyte/EC interactions per monolayer (F); $n=2$ experiments with one monolayer per condition for each experiment and at least 100 monocyte/EC interactions per monolayer (G). ${ }^{*} P<0.05,{ }^{*} P<0.01$. Scale bar $=10 \mu \mathrm{m}$. HUVEC, human umbilical vein endothelial cell; LBRC, lateral border recycling compartment; mAb, monoclonal antibody; PBMC, peripheral blood mononuclear cell; PECAM, platelet endothelial cell adhesion molecule; TEM, transendothelial migration; TR, targeted recycling.

of recycling LBRC around transmigrating leukocytes. One-pixel-wide lines were drawn along the area surrounding the site of diapedesis. The mean fluorescence intensity in contact with the leukocyte was divided by the average intensity of constitutively recycled PECAM at the adjacent cell border to calculate LBRC enrichment around transmigrating leukocytes. We typically examined multiple monolayers until we had examined at least 100 transmigration events for each variable tested. To avoid unintentional bias each leukocyte in contact with the endothelial border was scored, whether it had initiated TEM. Each experiment was repeated at least three times. For imaging, the cells plated on collagen gels in 96-well plates were transferred onto 35-mm glass-bottom dishes and imaged on a PerkinElmer UltraVIEW VoX Confocal Spinning Disk Microscope (PerkinElmer, Boston, MA), using appropriate filters for visualizing Alexa 488, Alexa 568, and Dylight 650 conjugated probes (Thermo Fisher Scientific). Images were acquired with a UPlanApo $40 \times$ oil immersion objective and Volocity software (PerkinElmer). Image processing and quantification of data were performed with Volocity version 6.2.1 and ImageJ version 1.46r $(\mathrm{NIH}$, Bethesda, MA) image processing software.

\section{Statistical Analysis}

All data were analyzed by pairwise comparison with the use of a two-tailed $t$-test assuming unequal variances, with the Bonferroni correction for multiple comparisons as appropriate.

\section{Results}

\section{Blocking Kinesin-1 with SUK4 Inhibits Paracellular TEM}

There are 45 KIF genes that encode 45 KHCs. There are multiple associated light chains in the human genome. ${ }^{24,25}$ Because we had a function-blocking monoclonal antibody (SUK4) against kinesin-1, we first focused on kinesin-1 as a likely candidate to mediate targeted recycling of the LBRC. Three genes, KIFA, KIFB, and KIFC, encode three variants of kinesin-1, differing only at their C-terminal ends (1028, 


\section{Resistant Kinesin-1 Construct}

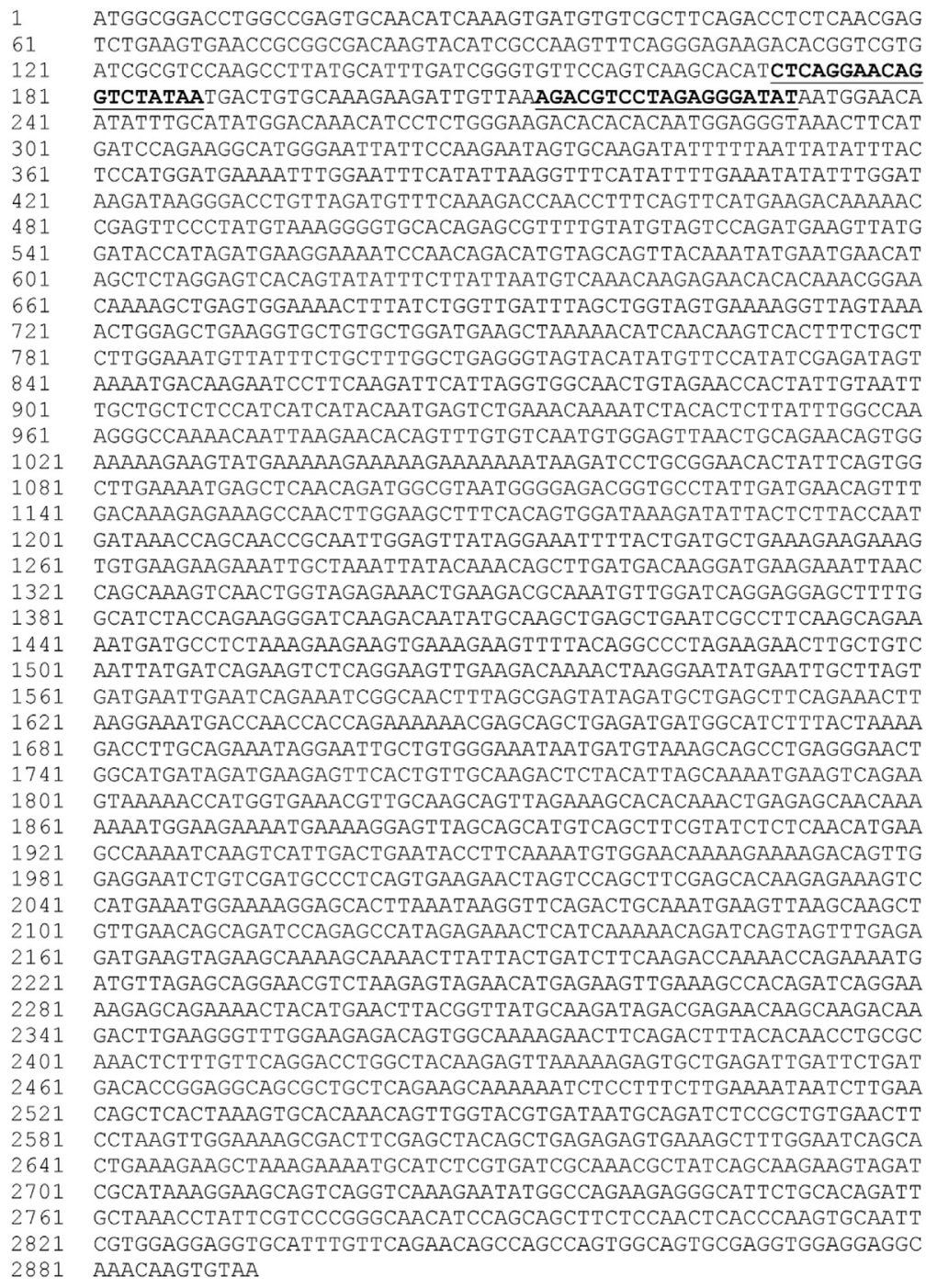

1 ATGGCGGACCTGGCCGAGTGCAACATCAAAGTGATGTGTCGCTTCAGACCTCTCAACGAG 61 TCTGAAGTGAACCGCGGCGACAAGTACATCGCCAAGTTTCAGGGAGAAGACACGGTCGTG 121 ATCGCGTCCAAGCCTTATGCATTTGATCGGGTGTTCCAGTCAAGCACAT CTCAGGAACAG 181 GTCTATAATGACTGTGCAAAGAAGATTGTTAAAGACGTCCTAGAGGGATATAATGGAACA 241 ATATTTGCATATGGACAAACATCCTCTGGGAAGACACACACAATGGAGGGTAAACTTCAT 301 GATCCAGAAGGCATGGGAATTATTCCAAGAATAGTGCAAGATATTTTTAATTATATTTAC 361 TCCATGGATGAAAATTGGAATTCATATTAAGGTTTCATATTTTGAAATATATTTGGAT 421 AAGATAAGGGACCTGTTAGATGTTTCAAAGACCAACCTTTCAGTTCATGAAGACAAAAAC 481 CGAGTTCCCTATGTAAAGGGGTGCACAGAGCGTTTTGTATGTAGTCCAGATGAAGTTATG 541 GATACCATAGATGAAGGAAAATCCAACAGACATGTAGCAGTTACAAATATGAATGAACAT 601 AGCTCTAGGAGTCACAGTATATTTCTTATTAATGTCAAACAAGAGAACACACAAACGGAA 661 CAAAAGCTGAGTGGAAAACTTTATCTGGTTGATTTAGCTGGTAGTGAAAAGGTTAGTAAA 721 ACTGGAGCTGAAGGTGCTGTGCTGGATGAAGCTAAAAACATCAACAAGTCACTTTCTGCT 781 CTTGGAAATGTTATTTCTGCTTTGGCTGAGGGTAGTACATATGTTCCATATCGAGATAGT 841 AAAATGACAAGAATCCTTCAAGATTCATTAGGTGGCAACTGTAGAACCACTATTGTAATT 901 TGCTGCTCTCCATCATCATACAATGAGTCTGAAACAAAATCTACACTCTTATTTGGCCAA 961 AGGGCCAAAACAATTAAGAACACAGTTTGTGTCAATGTGGAGTTAACTGCAGAACAGTGG 1021 AAAAAGAAGTATGAAAAAGAAAAAGAAAAAAATAAGATCCTGCGGAACACTATTCAGTGG 1081 CTTGAAAATGAGCTCAACAGATGGCGTAATGGGGAGACGGTGCCTATTGATGAACAGTTT 1141 GACAAAGAGAAAGCCAACTTGGAAGCTTTCACAGTGGATAAAGATATTACTCTTACCAAT 1201 GATAAACCAGCAACCGCAATTGGAGTTATAGGAAATTTTACTGATGCTGAAAGAAGAAAG 1261 TGTGAAGAAGAAATTGCTAAATTATACAAACAGCTTGATGACAAGGATGAAGAAATTAAC CAGCAAAGTCAACTGGTAGAGAAACTGAAGACGCAAATGTTGGATCAGGAGGAGCTTTTG 1381 GCATCTACCAGAAGGGATCAAGACAATATGCAAGCTGAGCTGAATCGCCTTCAAGCAGAI

1441 AATGATGCCTCTAAAGAAGAAGTGAAAGAAGTTTTACAGGCCCTAGAAGAACTTGCTGTC AATTATGATCAGAAGTCTCAGGAAGTTGAAGACAAAACTAAGGAATATGAATTGCTTAGT 1561 GATGAATTGAATCAGAAATCGGCAACTTTAGCGAGTATAGATGCTGAGCTTCAGAAACTT 1621 AAGGAAATGACCAACCACCAGAAAAAACGAGCAGCTGAGATGATGGCATCTTTACTAAAA 1681 GACCTTGCAGAAATAGGAATTGCTGTGGGAAATAATGATGTAAAGCAGCCTGAGGGAACT 1741 GGCATGATAGATGAAGAGTTCACTGTTGCAAGACTCTACATTAGCAAAATGAAGTCAGAA 1801 GTAAAAACCATGGTGAAACGTTGCAAGCAGTTAGAAAGCACACAAACTGAGAGCAACAAA AAAATGGAAGAAAATGAAAAGGAGTTAGCAGCATGTCAGCTTCGTATCTCTCAACATGA 1921 GCCAAAATCAAGTCATTGACTGAATACCTTCAAAATGTGGAACAAAAGAAAAGACAGTTG 1981 GAGGAATCTGTCGATGCCCTCAGTGAAGAACTAGTCCAGCTTCGAGCACAAGAGAAAGTC CATGAaATGAAAAGGAGCACTTAAATAAGGTTCAGACTGCAAATGAAGTTAAGCAAGCT 2101 GTTGAACAGCAGATCCAGAGCCATAGAGAAACTCATCAAAAACAGATCAGTAGTTTGAGA 2161 GATGAAGTAGAAGCAAAAGCAAAACTTATTACTGATCTTCAAGACCAAAACCAGAAAATG 2281 AAGAGCAGAAAACTACATGAACTTACGGTTATGCAAGATAGACGAGAACAAGCAAGACAA 2341 GACTTGAAGGGTTTGGAAGAGACAGTGGCAAAAGAACTTCAGACTTTACACAACCTGCGC AAACTCTTTGTTCAGGACCTGGCTACAAGAGTTAAAAAGAGTGCTGAGATTGATTCTGAT 2461 GACACCGGAGGCAGCGCTGCTCAGAAGCAAAAAATCTCCTTTCTTGAAAATAATCTTGAД 2521 CAGCTCACTAAAGTGCACAAACAGTTGGTACGTGATAATGCAGATCTCCGCTGTGAACTT CCTAAGTTGAAAAGCGACTTCGAGCTACAGCTGAGAGAGTGAAAGCTTTGGaATCAGCA 2641 CTGAAAGAAGCTAAAGAAAATGCATCTCGTGATCGCAAACGCTATCAGCAAGAAGTAGAT 2701 CGCATAAAGGAAGCAGTCAGGTCAAAGAATATGGCCAGAAGAGGGCATTCTGCACAGATT 2761 GCTAAACCTATTCGTCCCGGGCAACATCCAGCAGCTTCTCCAACTCACCCAAGTGCAATT 2881 AAACAAGTGTAA

962, and 956 amino acids, respectively). ${ }^{25,26}$ HUVECs express the ubiquitous KIF5B isoform and KIF5C but not KIF5A. ${ }^{6}$ To study the role of kinesin-1 in tem, we microinjected a function-blocking monoclonal antibody specific for Kinesin-1 (SUK4) that recognizes both KIF5B and KIF5C isoforms ${ }^{27,28}$ or isotype control mouse $\mathrm{IgG}$ into confluent HUVEC monolayers and conducted TEM assays. Unlike the HD anti-kinesin antibody that we used in the previous study, ${ }^{6}$ SUK4 is specific for kinesin-1 and does not recognize other members of the kinesin motor superfamily. ${ }^{27}$ Furthermore, although it was originally raised against sea urchin kinesin-1, it has been proven to be cross-reactive with mammalian kinesin-1. ${ }^{29-31}$

The microinjected monolayers remained intact, and monocytes were able to attach and migrate (Figure 1A). No difference was found in the ability of the monocytes to adhere to the mouse IgG-injected endothelial cells compared with those of the SUK4-injected endothelial cells (Figure 1B). Within 8 minutes approximately $50 \%$ of
Figure 2 Sequence of kinesin heavy chain rescue construct. Targeted sequences shown are mutated to be resistant to knockdown. The mutated regions are in bold and underlined. adherent monocytes were already undergoing TEM across HUVECs injected with isotype-matched mouse IgG. In contrast, transmigration was ablated in the SUK4-injected HUVECs (Figure 1C), suggesting a role for kinesin-1 in leukocyte TEM.

\section{Blocking Kinesin-1 with SUK4 Inhibits Targeted Recycling of the LBRC}

To further analyze the mechanism of kinesin-1 in paracellular TEM, targeted recycling assays were performed to determine whether kinesin-1 is necessary for targeting the LBRC to the site of TEM. The LBRC constitutively recycles evenly around the endothelial cell with a halftime of 10 minutes. When leukocytes transmigrate, even though constitutive recycling still occurs somewhat evenly along the cell borders, LBRC membrane in their vicinity preferentially traffics toward them (targeted recycling), resulting in enhanced fluorescence at the site of TEM in these 
A
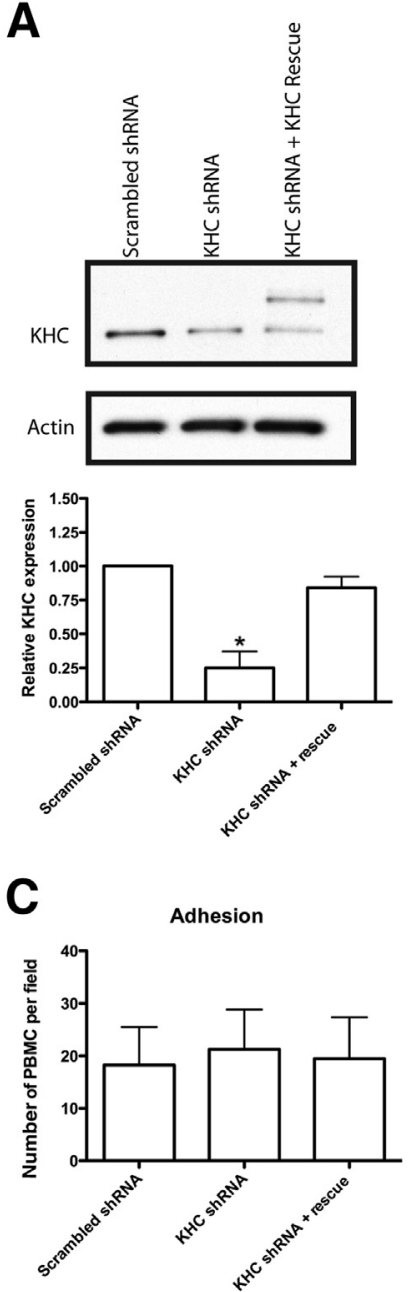

B
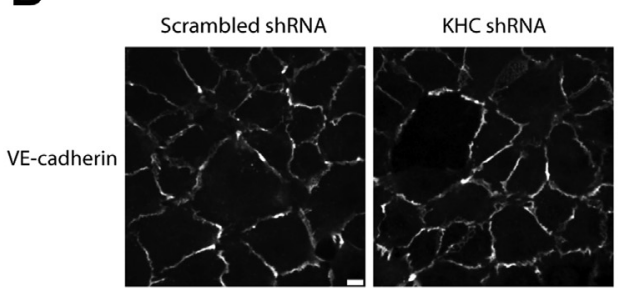

KHC shRNA + KHC rescue
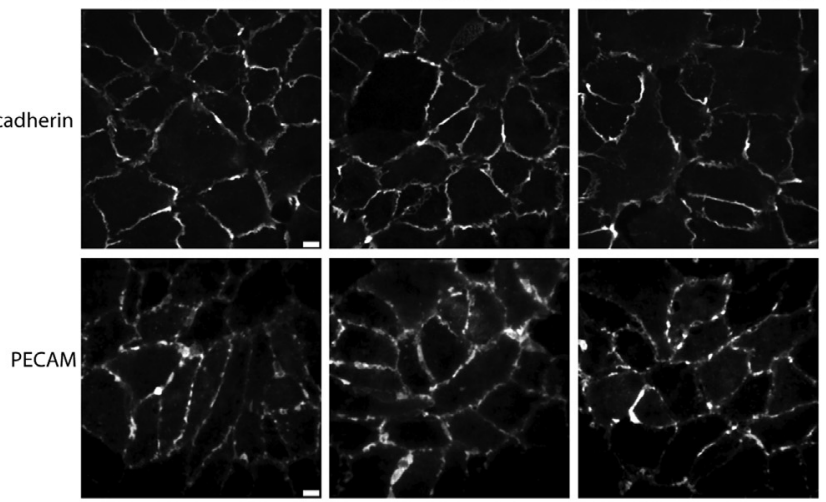

Microtubules
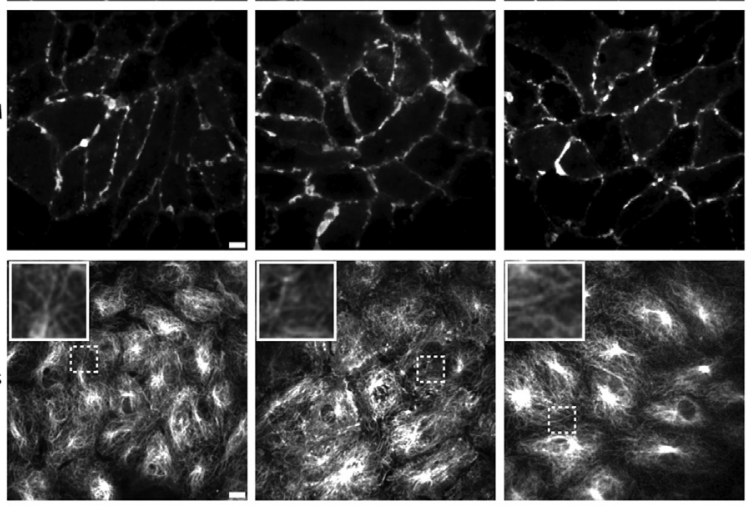

D
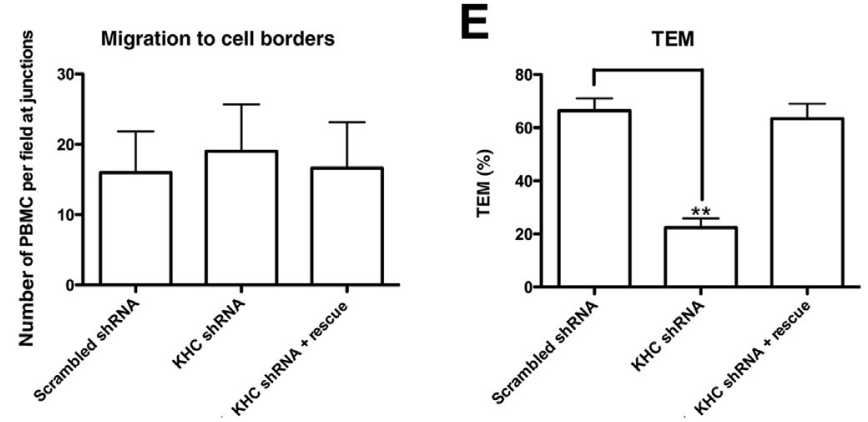

Figure 3 Knockdown of kinesin-1 blocks TEM. A: Western blot analysis was performed 72 hours after infection with scrambled shRNA, kinesin-1 shRNA (KHC shRNA), or kinesin-1 shRNA with addition of the kinesin-1 rescue construct tagged with mCherry (upper band in KHC shRNA + Rescue lane). A polyclonal antibody against kinesin-1 was used to probe for KHC. Quantification of the relative total KHC expression. B: After knockdown of kinesin-1, cells were stained for VE-cadherin, PECAM, and microtubules. Insets show enlargements of the area in dotted boxes. C and D: PBMCs were allowed to transmigrate on infected HUVEC monolayers for 8.5 minutes before quantifying adhesion (C) and migration to cell borders (D). E: PBMCs were allowed to transmigrate on HUVEC monolayers for 60 minutes to measure TEM. Data are expressed as means \pm SEM for all experiments. $n=3$ experiments each with three monolayers/variable per experiment with a total of $>600$ cell interactions counted $(\mathbf{C}-\mathbf{E}) .{ }^{*} P<0.05,{ }^{*} P<0.01$. Scale bar $=10 \mu \mathrm{m}$. Original magnification, $\times 312$ (insets). HUVEC, human umbilical vein endothelial cell; KHC, kinesin heavy chain; PBMC, peripheral blood mononuclear cell; PECAM, platelet-endothelial cell adhesion molecule; TEM, transendothelial migration; VE, vascular endothelial.

assays. ${ }^{5,6}$ In these experiments, transmigration is allowed to proceed for a short time ( 7 to 8 minutes) to observe the interaction of recycling LBRC with leukocytes as they transmigrate $^{5}$ and before fluorescence associated with constitutive recycling catches up.

We measured the average intensity of the recycled LBRC fluorescence around all of the monocytes at the junctions and compared it with the average intensity of constitutively recycled LBRC fluorescence along the neighboring junction (arbitrarily set at 1.0). To avoid unintentional bias, we quantified the intensity of recycling LBRC under or around every leukocyte situated at a junction (including about half of them that have not begun transmigration). This was expressed as LBRC enrichment and was a measure of the entire population of bound leukocytes. We observed a twofold average enrichment of the LBRC in contact with transmigrating peripheral blood mononuclear cells in the mouse IgG isotype control-injected HUVECs. Generally, this appeared as a ring or crescent around the transmigrating leukocyte, but sometimes as small patches where the leukocyte engages the EC border (Figure 1D). In contrast, HUVECs microinjected with the SUK4 mAb did not support TEM (Figure 1B) and showed no increase in recycling of the LBRC associated with leukocytes adherent to their cell borders (Figure 1, D and E). The percentage of monocytes associated with enhanced LBRC recycling ( $\geq$ twofold compared with adjacent junctions) was denoted as percent targeted recycling. At this early time point only about $50 \%$ of the monocytes were undergoing TEM in control co-cultures, but virtually all of them were associated with enhanced 
A
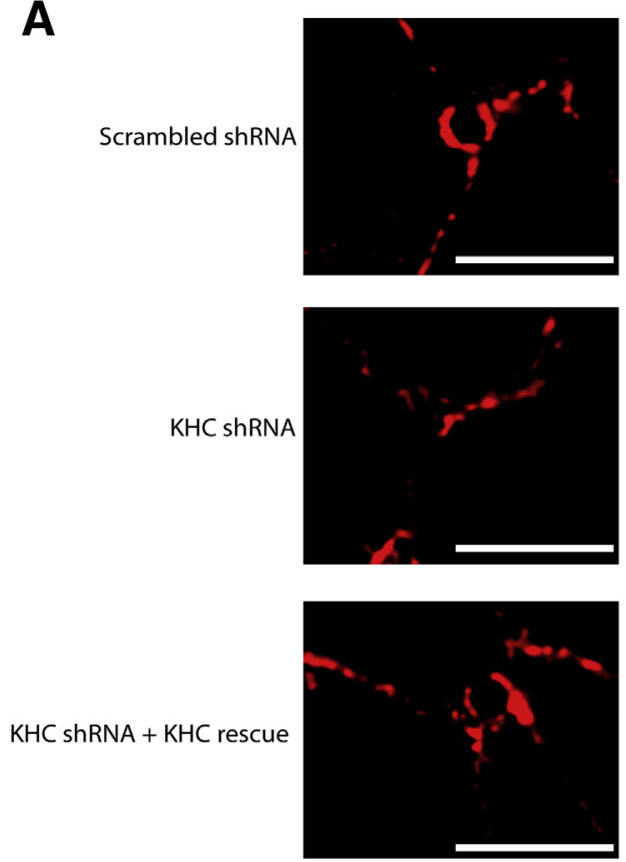
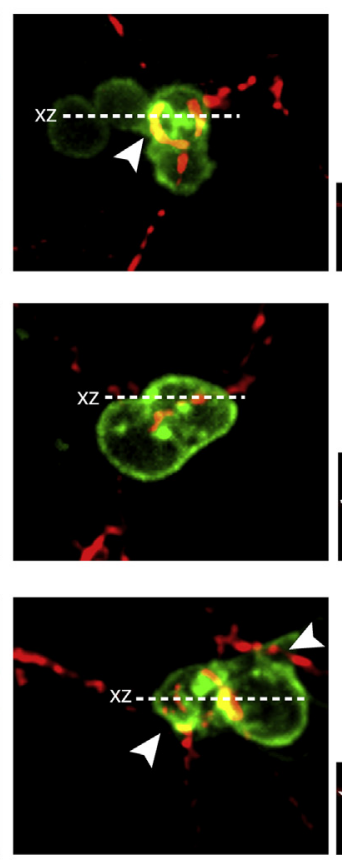

B
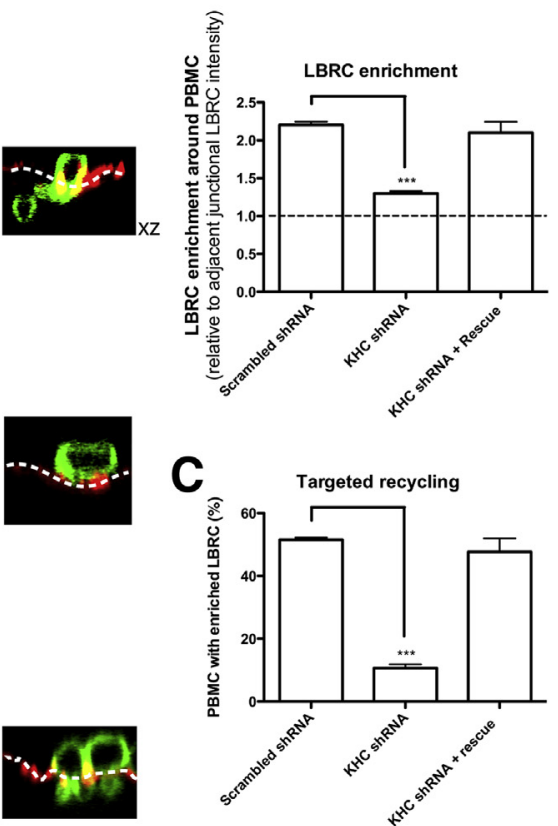

Figure 4 Knockdown of kinesin-1 in HUVECs results in a decrease in LBRC targeted recycling. HUVECs were transduced with scrambled shRNA, kinesin-1 shRNA (KHC shRNA), or kinesin-1 shRNA with addition of the kinesin-1 rescue construct (KHC shRNA + Rescue). Monocytes were allowed to transmigrate for 8.5 minutes. A: Confocal stacks were imaged with CD18 in green and recycled LBRC in red. Orthogonal projections (xz) are depicted as smaller images to the right of their corresponding images and indicate whether the monocyte is in the process of TEM or engaging the endothelial cell border. Arrowheads indicate site of leukocyte TEM. Dotted lines in the orthogonal projection indicate abluminal surface of endothelial cells. Constitutive recycling occurs, including under the blocked leukocyte, ${ }^{5,6}$ when kinesin- 1 is knocked down, but there is no enrichment of LBRC around the monocyte. The KHC shRNA + Rescue panel shows two transmigrating monocytes, one exhibiting a ring of enrichment surrounding it and the other exhibiting local enrichment at both sides of it. B: LBRC enrichment was measured around all adherent monocytes. The dotted line represents no change in LBRC enrichment relative to adjacent junctional LBRC intensity. C: Targeted recycling significantly decreased on knockdown of KLC1 and was restored to control levels with the rescue. Data are expressed as means \pm SEM. $n=3$ experiments with three monolayers per condition per experiment and $>300$ monocyte/endothelial cell interactions per experiment (B and C). ${ }^{* *} P<0.001$. Scale bar $=10 \mu \mathrm{m}$. HUVEC, human umbilical vein endothelial cell; $\mathrm{KHC}$, kinesin heavy chain; LBRC, lateral border recycling compartment; PBMC, peripheral blood mononuclear cell; TEM, transendothelial migration.

trafficking of LBRC membrane (targeted recycling) (Figure 1F). Targeted recycling was ablated when HUVECs were microinjected with SUK4 (Figure 1F), suggesting that kinesin-1 was required for targeting the LBRC to the site of TEM. As an additional control for specificity, we microinjected K2.4, a monoclonal antibody against kinesin- $2,{ }^{32}$ in parallel with SUK4 and isotype control. Only SUK4 blocked TEM (Figure 1G).

\section{shRNA Knockdown of Kinesin-1 Inhibits Paracellular TEM}

In a complementary approach, we specifically knocked down kinesin-1 in endothelial cells. There are three genes for kinesin-1 in mammals: KIF5A, KIF5B, and KIF5C. ${ }^{24,25}$ Because KIF5A is not expressed in HUVECs and KIF5C was detected by PCR, but not immunofluorescence, we focused on $K I F 5 B$, which is strongly expressed in HUVECs. ${ }^{6}$ Two KIF5B shRNA knockdown constructs were cloned into destination vectors for adenoviral expression. Other than a slight overlap, the target sequences for KIF5B did not match sites on KIF5A or KIF5C sequences. The amplified virus was used to transduce HUVECs. ${ }^{33,34}$
Western blot analysis of virally transduced HUVEC was used to quantify knockdown of kinesin-1. A 75\% knockdown of kinesin-1 was measured 72 hours after infection with the shRNA-expressing adenovirus, and knockdown was rescued after addition of the wild-type kinesin-1 adenovirus construct that was tagged with mCherry to distinguish it from residual endogenous kinesin-1 and mutated to prevent it from being targeted by the kinesin-1 shRNA (Figures 2 and 3A). Knockdown of kinesin-1 did not affect the distribution of microtubules or the expression levels or distribution of vascular endothelial-cadherin or PECAM (Figure 3B).

Knockdown of kinesin- 1 in ECs did not affect the number of monocytes attached to HUVEC monolayers, showing that knocking down kinesin-1 in HUVECs did not affect adhesion of monocytes before TEM (Figure 3C). Likewise, there was also no difference in the average number of monocytes at HUVEC junctions between control and knockdown cells, suggesting that kinesin-1 is not required for migration of monocytes to cell borders before TEM (Figure 3D). However, knockdown of kinesin-1 in HUVECs resulted in a significant decrease in transmigration of monocytes in our standard 1-hour TEM assay, supporting 
A

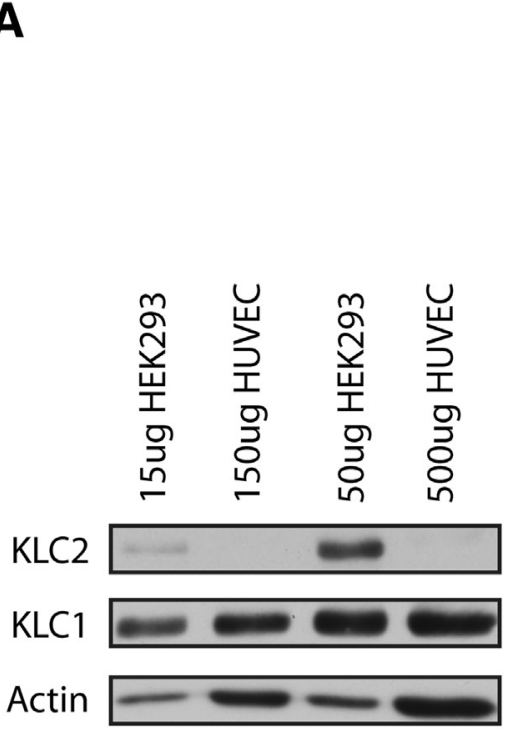

B

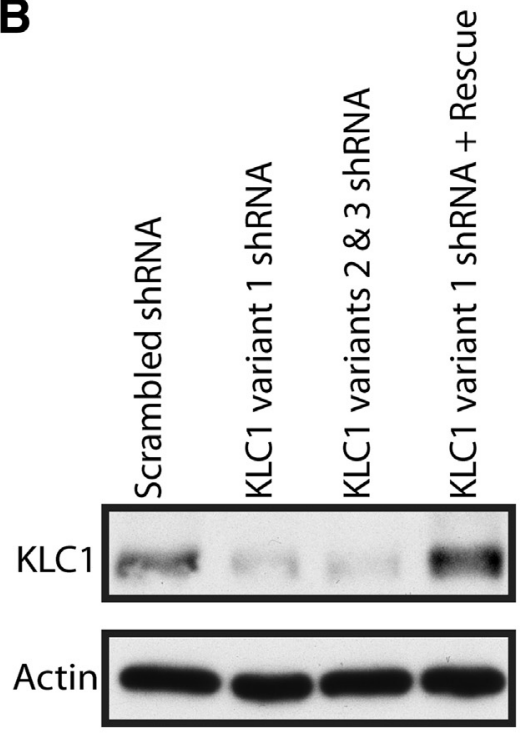

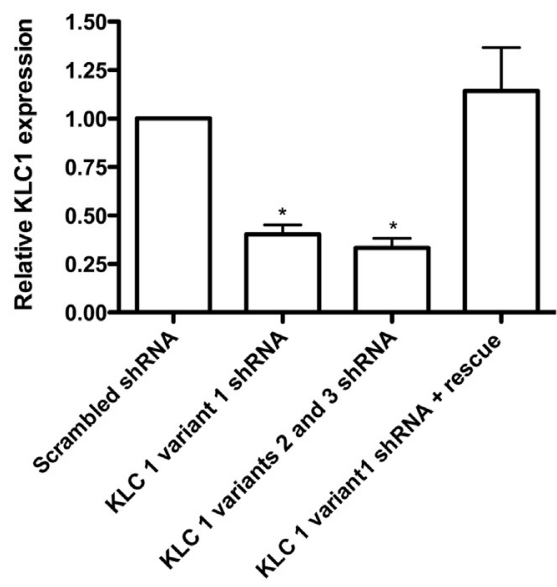

F
C

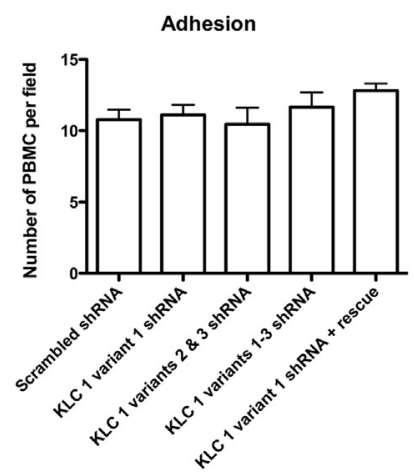

D

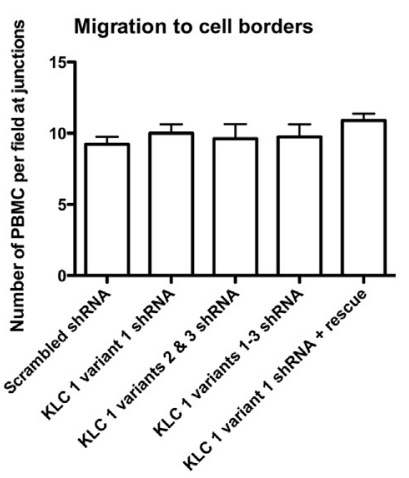

E

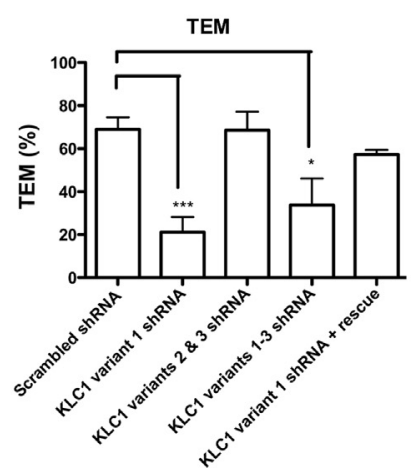

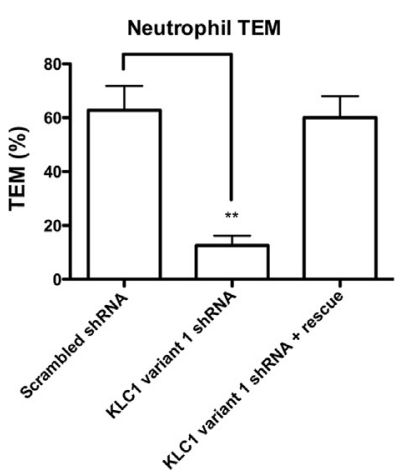

Figure 5 Knockdown of KLC1 isoform variant 1 in HUVEC blocks TEM. A: Western blot analysis of KLC2 expression in HEK293 cells versus HUVECs demonstrates absence of KLC2 in HUVECs. Cells were plated on Mattek dishes and lyzed 96 hours later. The amount of lysate added to the gels is indicated and was adjusted to obtain similar KLC1 expression levels in 293 cells versus HUVECs to fairly assess KLC2 levels in 293 cells versus HUVEC. B: HUVECs were transduced with scrambled shRNA, KLC1 variant 1 shRNA, KLC1 variants 2 and 3 shRNA, or KLC1 variant 1 shRNA and the KLC1 variant 1 rescue construct. Cells were lyzed 72 hours later. Quantification of the Western blot analysis. C and D: HUVECs were transduced with scrambled shRNA, KLC1 variant 1 shRNA, KLC1 variants 2 and 3 shRNA, KLC variants 1 to 3 shRNA, or KLC1 variant 1 shRNA with the KLC1 variant 1 rescue construct. Adhesion (C) and migration (D) to cell borders were measured after an 8.5-minute TEM period. Monocytes (E) or neutrophils (F) were allowed to transmigrate for 60 minutes before TEM was quantified. Data are expressed as means \pm SEM. $n=3$ experiments $(\mathbf{B}) ; \mathrm{n}=3$ experiments with three monolayers per condition per experiment $(\mathbf{C}-\mathbf{F})$. ${ }^{*} P<0.05,{ }^{*} P<0.01$, and $* * * P<0.001$. HUVEC, human umbilical vein endothelial cell; KLC, kinesin light chain; PBMC, peripheral blood mononuclear cell; TEM, transendothelial migration.

our microinjection results. TEM was rescued on transduction with the kinesin-1 rescue construct (Figure 3E).

\section{shRNA Knockdown of Kinesin-1 Inhibits Targeted Recycling of the LBRC}

Next, we studied the effect of KHC knockdown on targeted recycling. In cells transduced with scrambled shRNA, membrane recycled from the LBRC, as expected, and was detected as a rim of enriched fluorescence around monocytes caught in the act of TEM (Figure 4A). On knockdown of kinesin-1 in HUVECs, concomitant with the block in TEM, there was virtually no enrichment of the LBRC surrounding monocytes at endothelial junctions, although constitutive recycling occurred under the blocked monocytes (Figure 4A) as seen previously. ${ }^{5,6}$ Re-expression of kinesin-1 with a rescue cDNA construct restored targeted recycling to control levels (Figure 4B). Note that in this experiment recycling LBRC was labeled with Alexa 488 to avoid interference by the mCherry tag of the rescue construct. The figure has been pseudocolored for consistency with the other figures. Therefore, targeted recycling of the LBRC was significantly decreased on knockdown of kinesin-1 (Figure 4C). Together, these data suggest that Kinesin-1 function was required for targeted recycling and, hence, TEM. 
A

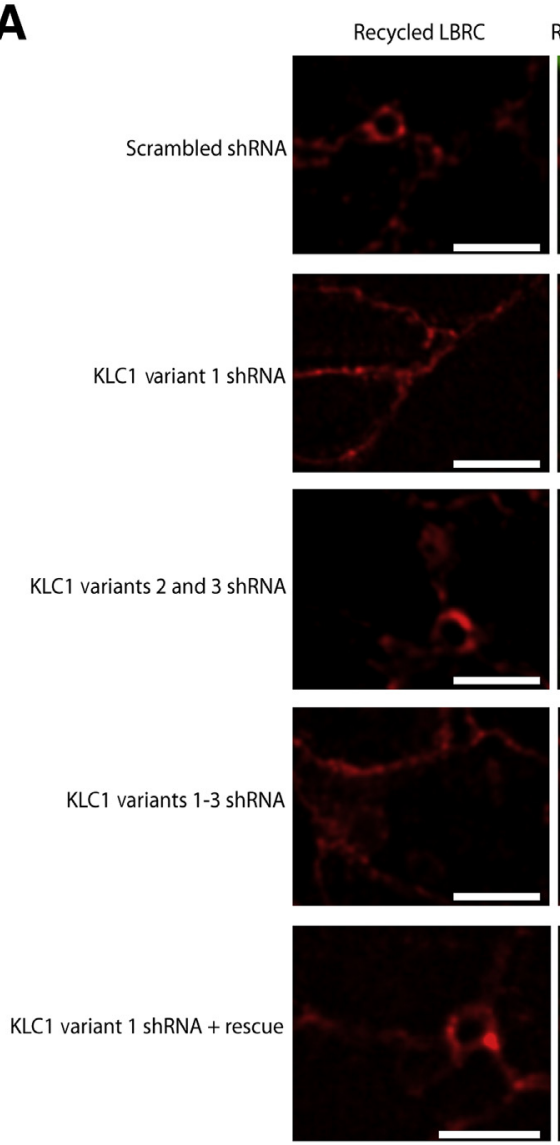

Recycled LBRC with monocyte
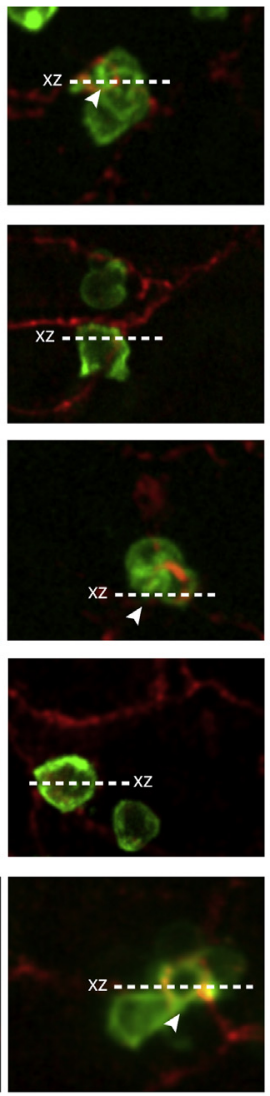
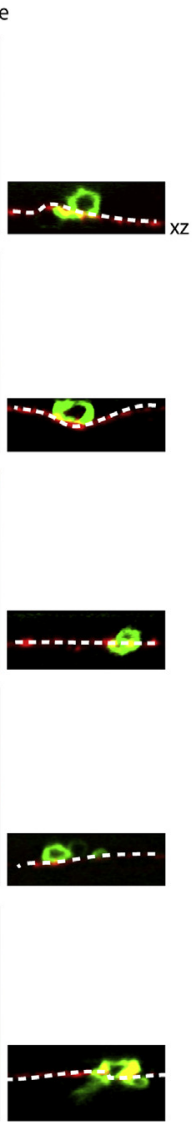

B
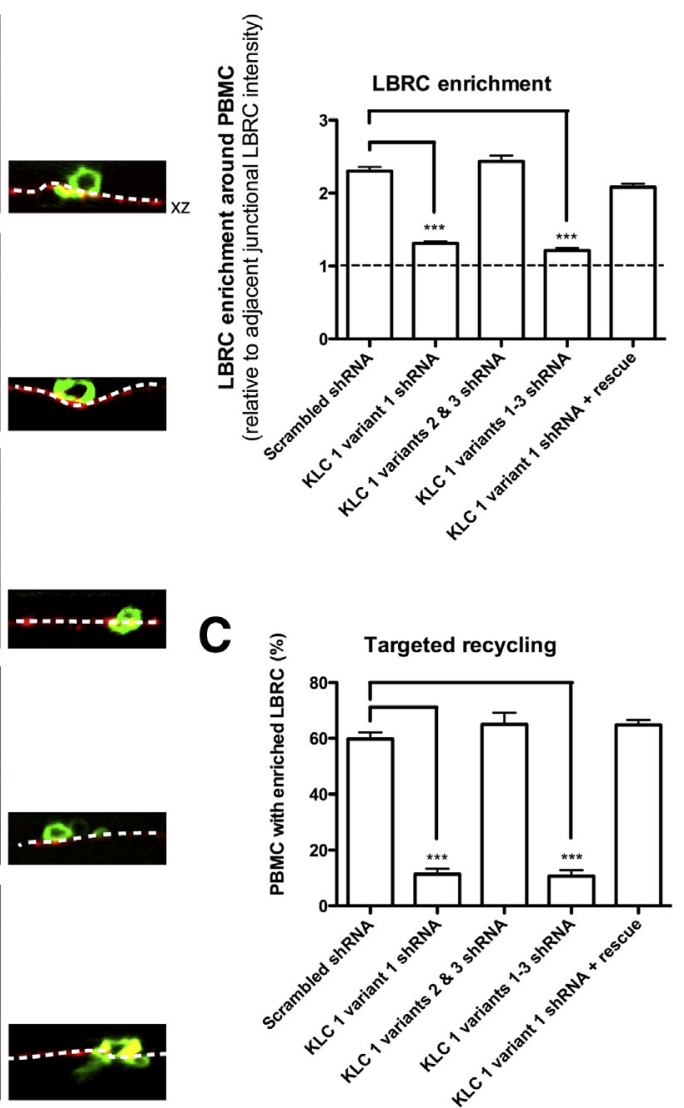

Figure 6 Knockdown of KLC1 isoform variant 1 in HUVECs results in a decrease in LBRC targeted recycling. HUVECs were transduced with scrambled shRNA, KLC1 variant 1 shRNA, KLC1 variants 2 and 3 shRNA, and KLC variants 1 to 3 shRNA. A and B: Monocytes were allowed to transmigrate for 8.5 minutes. Confocal stacks were imaged (A), with their corresponding orthogonal projections ( $\mathrm{xz}$ ) as smaller images to the right, and LBRC enrichment was measured around all adherent monocytes (B). The dotted line represents no change in LBRC enrichment. C: Targeted recycling significantly decreased on knockdown of KLC1 isoform variant 1 . Arrowheads indicate site of leukocyte transendothelial migration. Dotted lines in the orthogonal projection indicate abluminal surface of endothelial cells. Data are expressed as means \pm SEM. $n=3$ experiments with three monolayers per condition per experiment (B and $\mathbf{C}) .{ }^{* *} P<0.001$. Scale bar $=10 \mu \mathrm{m}$. HUVEC, human umbilical vein endothelial cell; KLC, kinesin light chain; LBRC, lateral border recycling compartment; PBMC, peripheral blood mononuclear cell.

\section{Knockdown of KLC1 Variant 1 Inhibits TEM}

We then looked at the potential for KLC involvement in mediating binding between the LBRC and kinesin-1, specifically during targeted recycling and TEM. Although at least four kinesin-1 light chains have been described or predicted, ${ }^{35,36}$ KLC1 and KLC2 are the two that have been reported in the literature to interact with kinesin- 1 and can mediate cargo binding. ${ }^{35}$ However, KLC2 was not expressed by HUVECs (Figure 5A), ${ }^{6}$ so we focused our attention on KLC1. KLC1 has several well-described potential splice variants. ${ }^{37,38}$ We transduced HUVECs with adenovirus-expressing shRNA that target the most commonly reported specific splice variants of human $\mathrm{KLC} 1$ and measured a decrease in KLC1 expression compared with HUVECs transduced with scrambled shRNA (Figure 5B). The hairpins transduced targeted variant 1 (KLC1C), variants $2(\mathrm{KLC} 1 \mathrm{H})$, and 3 (KLC1D), or all three variants.

Neither adhesion (Figure 5C) nor migration (Figure 5D) to cell borders was affected on transduction of any
KLC shRNA construct. TEM experiments were then conducted to assess any involvement of KLC1 in this process. Interestingly, TEM was only reduced on transduction with the KLC1 variant 1 shRNA construct and the shRNA simultaneously targeting variants 1,2 , and 3 but not the construct targeting just KLC1 variants 2 and 3. TEM is restored on transduction with virus expressing KLC1 variant $1 \mathrm{cDNA}$. These data demonstrate that KLC1 variant 1 is required for TEM (Figure 5, E and F).

\section{Knockdown of KLC1C Inhibits Targeted Recycling of the LBRC}

We next looked for a possible role of KLC1C in targeted recycling. On knockdown of KLC1C in HUVECs, overall LBRC enrichment around and under adherent monocytes (Figure 6, A and B) and the percentage of monocytes around which LBRC is enriched (targeted recycling, Figure 6C) were significantly diminished but were restored to control levels on rescue of this specific KLC1 variant. Knockdown 
of the other KLC1 isoform variants did not affect the level of targeted recycling.

\section{Discussion}

We report in this study that endothelial cell kinesin- 1 is the $\mathrm{KHC}$ involved in transporting the LBRC to the site of TEM (targeted recycling) and thereby supporting TEM. Knocking down kinesin-1 in endothelial cells or microinjecting a function-blocking monoclonal antibody specific for kinesin-1 into endothelial cells blocked targeted recycling and TEM. Perhaps more important, we found that KLC1, and more specifically $\mathrm{KLC} 1 \mathrm{C}$, is required for targeted recycling of the LBRC and TEM. Because KLCs mediate the binding of cargo to $\mathrm{KHCs}$, this suggests that $\mathrm{KLC1C}$ is responsible for the binding of the LBRC to kinesin-1 during targeted recycling and TEM.

To narrow our focus on which kinesin was responsible for moving the LBRC during targeted recycling, we first determined which ones were expressed in endothelial cells. With the use of RT-PCR, we were able to detect expression of 23 of the 45 known kinesin genes. ${ }^{6}$ After eliminating the mitotic kinesins (which function during cytokinesis, chromosome segregation, and microtubule destabilization) and minus-end motors (because paracellular migration would require plus-end movement to the site of TEM at endothelial junctions) 13 candidates still remained that were capable of moving membrane organelles along microtubules in the direction of the plus end. Because we had a functionblocking monoclonal antibody (SUK4) against kinesin-1, we started there. HUVECs express $K I F 5 B$ and $K I F 5 C^{6}$; SUK4 recognizes both gene products. ${ }^{28}$ We used similar logic to determine which KLCs to target first.

We did not knock down and rescue every KHC and KLC expressed in endothelial cells. Therefore, we cannot completely rule out that other KHCs or KLCs may play a role in the movement of the LBRC in targeted recycling. However, because we blocked targeted recycling and TEM by $75 \%$ to $>95 \%$ in our assays by interfering with kinesin-1 or $\mathrm{KLC} 1 \mathrm{C}$, we can say that these proteins are critical for targeted recycling and TEM. Because $K I F 5 B$ is ubiquitously expressed, and knocking it down has such a large effect on targeted recycling of the LBRC and TEM, the effect of knocking down other $\mathrm{KHCs}$ or even other kinesin-1 isoforms in the presence of endogenous $K I F 5 B$ would be difficult to detect experimentally. If other KHCs or KLCs are involved, they either play a minor role or depend on kinesin-1 and KLC1C for this function. Furthermore, microinjecting K2.4 mAb against kinesin-2 in HUVECs did not affect TEM levels, further supporting our hypothesis for kinesin-1 as the responsible motor protein for trafficking the LBRC during leukocyte TEM.

Our TEM assay is an end point assay in which we can observe leukocytes after 1 hour of allowing them to migrate across endothelial cell junctions. This demonstrates that knockdown of kinesin-1 or KLC1 variant 1 does not merely slow down TEM, it inhibits it. Our targeted recycling assay is meant to catch leukocytes in the act of transmigration (approximately 8 minutes of migration). Catching leukocytes during migration gives us the opportunity to observe enrichment of the LBRC around migrating leukocytes. These assays are performed and completed within a few hours of microinjection, so the effect of blocking kinesin-1 function on other physiologic processes is minimal. Furthermore, control cells are injected with an equal volume and concentration of control rabbit IgG. Notably, interference with kinesin-1 or KLC1C had no effect on adhesion of monocytes to the endothelial monolayers or migration of monocytes to the endothelial cell borders (Figures 1, 3, and 5). It was specific for inhibiting targeted recycling and TEM, as was depolymerization of microtubules by chemical means and microinjection of HD polyclonal antibody. ${ }^{6}$ Moreover, interfering with kinesin function has no effect on constitutive recycling of the LBRC $^{6}$; kinesin-1 and KLC1C disruption only affects targeted recycling of the LBRC to the site of TEM.

KLCs are composed of an $\alpha$-helical coiled-coil heptad repeat domain that binds to the $\mathrm{KHC}$, six imperfect tetratricopeptide repeats that mediate cargo binding ${ }^{39-42}$ and a variable C-terminal domain. ${ }^{38}$ Some cargoes may actually bind directly to the $\mathrm{KHC}$, such as milton, which competes with light chains for binding to the heavy chain. ${ }^{16,17}$ Because of alternative splicing in this C-terminal domain, different $\mathrm{KLC} 1$ variants have been proposed to mediate binding to specific cargoes such as vimentin, mitochondria, and Golgi membranes. ${ }^{37,43-45}$ Specifically, human KLC1 isoform variant $\mathrm{E}$ has been suggested to play a role in intracellular trafficking in amyloid- $\beta$ accumulation. ${ }^{46-48}$ Amyloid- $\beta$ accumulation is typical of Alzheimer disease, and knocking down KLC1E in neuroblastoma cells decreased the levels of amyloid- $\beta$. Conversely, overexpression of this variant increased amyloid- $\beta$ levels.

Our data show that KLC1C is required for targeted recycling and TEM. KHCs process along microtubules that carry bound cargo in the direction of the plus end. KLCs bind a restricted range of cargoes and tether them to KHCs by binding to the carboxy terminal of the heavy chains. Thus, our data suggest that KLC1C is the link between kinesin-1 and the LBRC cargo during targeted recycling.

This work helps to further elucidate the pathways responsible for the inflammatory response, which is of utmost importance in the development of future therapeutics for the many devastating diseases caused by inflammation. KLC1 variant 1 differs from the other KLC1 splice variants by 10 amino acids at its carboxy terminus. The specificity of KLC1 variant 1 for trafficking the LBRC to sites of TEM make it a potential target for anti-inflammatory therapy. Identifying the ligand(s) on the LBRC that bind to KLC1 variant 1 and how this binding is regulated will be the subject of future investigations. 


\section{Acknowledgments}

We thank Dr. Vladimir Gelfand (Northwestern University) for the SUK4 hybridoma cell line and K2.4 ascites, advice, and critical reading of the manuscript; Cliff Carpenter for excellent technical assistance; and Satya Khoun for training in microinjection. DNA sequencing was performed by the Feinberg School of Medicine Genomics Core; microinjections were performed in the Feinberg School of Medicine Center for Advanced Microscopy.

B.F.C. performed all of the experiments and wrote the first draft of the manuscript and W.A.M. supervised the project and edited the manuscript.

\section{References}

1. Butcher EC: Leukocyte-endothelial cell recognition: three (or more) steps to specificity and diversity. Cell 1991, 67:1033-1036

2. Ley K, Laudanna C, Cybulsky MI, Nourshargh S: Getting to the site of inflammation: the leukocyte adhesion cascade updated. Nat Rev Immunol 2007, 7:678-689

3. Muller WA: Mechanisms of leukocyte transendothelial migration. Annu Rev Pathol 2011, 6:323-344

4. Zen K, Parkos CA: Leukocyte-epithelial interactions. Curr Opin Cell Biol 2003, 15:557-564

5. Mamdouh Z, Chen X, Pierini LM, Maxfield FR, Muller WA: Targeted recycling of PECAM from endothelial cell surface-connected compartments during diapedesis. Nature 2003, 421:748-753

6. Mamdouh Z, Kreitzer GE, Muller WA: Leukocyte transmigration requires kinesin-mediated microtubule-dependent membrane trafficking from the lateral border recycling compartment. J Exp Med 2008, 205:951-966

7. Feng D, Nagy JA, Hipp J, Dvorak HF, Dvorak AM: Vesiculo-vacuolar organelles and the regulation of venule permeability to macromolecules by vascular permeability factor, histamine, and serotonin. J Exp Med 1996, 183:1981-1986

8. Sullivan DP, Muller WA: Neutrophil and monocyte recruitment by PECAM, CD99, and other molecules via the LBRC. Semin Immunopathol 2014, 36:193-209

9. Feng G, Sullivan DP, Han F, Muller WA: Segregation of VE-cadherin from the LBRC depends on the ectodomain sequence required for homophilic adhesion. J Cell Sci 2015, 128:576-588

10. Sullivan DP, Seidman MA, Muller WA: Poliovirus receptor (CD155) regulates a step in transendothelial migration between PECAM and CD99. Am J Pathol 2013, 182:1031-1042

11. Mamdouh Z, Mikhailov A, Muller WA: Transcellular migration of leukocytes is mediated by the endothelial lateral border recycling compartment. J Exp Med 2009, 206:2795-2808

12. Rodionov VI, Gyoeva FK, Gelfand VI: Kinesin is responsible for centrifugal movement of pigment granules in melanophores. Proc Natl Acad Sci U S A 1991, 88:4956-4960

13. Bloom GS, Wagner MC, Pfister KK, Brady ST: Native structure and physical properties of bovine brain kinesin and identification of the ATP-binding subunit polypeptide. Biochemistry 1988, 27:3409-3416

14. Hirokawa N, Pfister KK, Yorifuji H, Wagner MC, Brady ST, Bloom GS: Submolecular domains of bovine brain kinesin identified by electron microscopy and monoclonal antibody decoration. Cell $1989,56: 867-878$

15. Gindhart JG Jr, Desai CJ, Beushausen S, Zinn K, Goldstein LS: Kinesin light chains are essential for axonal transport in Drosophila. J Cell Biol 1998, 141:443-454

16. Glater EE, Megeath LJ, Stowers RS, Schwarz TL: Axonal transport of mitochondria requires milton to recruit kinesin heavy chain and is light chain independent. J Cell Biol 2006, 173:545-557
17. Rice SE, Gelfand VI: Paradigm lost: milton connects kinesin heavy chain to miro on mitochondria. J Cell Biol 2006, 173:459-461

18. Muller WA, Ratti CM, McDonnell SL, Cohn ZA: A human endothelial cell-restricted, externally disposed plasmalemmal protein enriched in intercellular junctions. J Exp Med 1989, 170:399-414

19. Muller WA, Weigl SA: Monocyte-selective transendothelial migration: dissection of the binding and transmigration phases by an in vitro assay. J Exp Med 1992, 176:819-828

20. Wright SD, Rao PE, Van Voorhis WC, Craigmyle LS, Iida K, Talle MA, Westberg EF, Goldstein G, Silverstein SC: Identification of the C3bi receptor of human monocytes and macrophages by using monoclonal antibodies. Proc Natl Acad Sci U S A 1983, 80: 5699-5703

21. Muller WA, Weigl SA, Deng X, Phillips DM: PECAM-1 is required for transendothelial migration of leukocytes. J Exp Med 1993, 178: 449-460

22. Anisman H, Baines MG, Berczi I, Bernstein CN, Blennerhassett MG, Gorczynski RM, Greenberg AH, Kisil FT, Mathison RD, Nagy E, Nance DM, Perdue MH, Pomerantz DK, Sabbadini ER, Stanisz A, Warrington RJ: Neuroimmune mechanisms in health and disease: 2. Disease. CMAJ 1996, 155:1075-1082

23. Liao F, Huynh HK, Eiroa A, Greene T, Polizzi E, Muller WA: Migration of monocytes across endothelium and passage through extracellular matrix involve separate molecular domains of PECAM-1. J Exp Med 1995, 182:1337-1343

24. Hirokawa N, Noda Y, Tanaka Y, Niwa S: Kinesin superfamily motor proteins and intracellular transport. Nat Rev Mol Cell Biol 2009, 10: 682-696

25. Lawrence CJ, Dawe RK, Christie KR, Cleveland DW, Dawson SC, Endow SA, Goldstein LS, Goodson HV, Hirokawa N, Howard J, Malmberg RL, McIntosh JR, Miki H, Mitchison TJ, Okada Y, Reddy AS, Saxton WM, Schliwa M, Scholey JM, Vale RD, Walczak CE, Wordeman L: A standardized kinesin nomenclature. J Cell Biol 2004, 167:19-22

26. Hirokawa N: From electron microscopy to molecular cell biology, molecular genetics and structural biology: intracellular transport and kinesin superfamily proteins, KIFs: genes, structure, dynamics and functions. J Electron Microsc (Tokyo) 2011, 60(Suppl 1):S63-S92

27. Ingold AL, Cohn SA, Scholey JM: Inhibition of kinesin-driven microtubule motility by monoclonal antibodies to kinesin heavy chains. J Cell Biol 1988, 107:2657-2667

28. Kanai Y, Okada Y, Tanaka Y, Harada A, Terada S, Hirokawa N: KIF5C, a novel neuronal kinesin enriched in motor neurons. J Neurosci 2000, 20:6374-6384

29. Jaulin F, Xue X, Rodriguez-Boulan E, Kreitzer G: Polarizationdependent selective transport to the apical membrane by KIF5B in MDCK cells. Dev Cell 2007, 13:511-522

30. Krylyshkina O, Kaverina I, Kranewitter W, Steffen W, Alonso MC, Cross RA, Small JV: Modulation of substrate adhesion dynamics via microtubule targeting requires kinesin-1. J Cell Biol 2002, 156: 349-359

31. Daire V, Giustiniani J, Leroy-Gori I, Quesnoit M, Drevensek S, Dimitrov A, Perez F, Pous C: Kinesin-1 regulates microtubule dynamics via a c-Jun N-terminal kinase-dependent mechanism. J Biol Chem 2009, 284:31992-32001

32. Cole DG, Chinn SW, Wedaman KP, Hall K, Vuong T, Scholey JM: Novel heterotrimeric kinesin-related protein purified from sea urchin eggs. Nature 1993, 366:268-270

33. Aizawa H, Sekine Y, Takemura R, Zhang Z, Nangaku M, Hirokawa N: Kinesin family in murine central nervous system. J Cell Biol 1992, 119:1287-1296

34. Niclas J, Navone F, Hom-Booher N, Vale RD: Cloning and localization of a conventional kinesin motor expressed exclusively in neurons. Neuron 1994, 12:1059-1072

35. Rahman A, Friedman DS, Goldstein LS: Two kinesin light chain genes in mice. Identification and characterization of the encoded proteins. J Biol Chem 1998, 273:15395-15403 
36. Junco A, Bhullar B, Tarnasky HA, van der Hoorn FA: Kinesin lightchain KLC3 expression in testis is restricted to spermatids. Biol Reprod 2001, 64:1320-1330

37. Khodjakov A, Lizunova EM, Minin AA, Koonce MP, Gyoeva FK: A specific light chain of kinesin associates with mitochondria in cultured cells. Mol Biol Cell 1998, 9:333-343

38. McCart AE, Mahony D, Rothnagel JA: Alternatively spliced products of the human kinesin light chain 1 (KNS2) gene. Traffic 2003, 4:576-580

39. Diefenbach RJ, Mackay JP, Armati PJ, Cunningham AL: The C-terminal region of the stalk domain of ubiquitous human kinesin heavy chain contains the binding site for kinesin light chain. Biochemistry 1998, 37:16663-16670

40. Cyr JL, Pfister KK, Bloom GS, Slaughter CA, Brady ST: Molecular genetics of kinesin light chains: generation of isoforms by alternative splicing. Proc Natl Acad Sci U S A 1991, 88:10114-10118

41. Wedaman KP, Knight AE, Kendrick-Jones J, Scholey JM: Sequences of sea urchin kinesin light chain isoforms. J Mol Biol 1993, 231:155-158

42. Gindhart JG Jr, Goldstein LS: Tetratrico peptide repeats are present in the kinesin light chain. Trends Biochem Sci 1996, 21:52-53

43. Gyoeva FK, Bybikova EM, Minin AA: An isoform of kinesin light chain specific for the Golgi complex. J Cell Sci 2000, 113(Pt 11):2047-2054
44. Woźniak MJ, Allan VJ: Cargo selection by specific kinesin light chain 1 isoforms. EMBO J 2006, 25:5457-5468

45. Liao G, Gundersen GG: Kinesin is a candidate for cross-bridging microtubules and intermediate filaments. Selective binding of kinesin to detyrosinated tubulin and vimentin. J Biol Chem 1998, 273: 9797-9803

46. Morihara T, Hayashi N, Yokokoji M, Akatsu H, Silverman MA, Kimura N, Sato M, Saito Y, Suzuki T, Yanagida K, Kodama TS, Tanaka T, Okochi M, Tagami S, Kazui H, Kudo T, Hashimoto R, Itoh N, Nishitomi K, Yamaguchi-Kabata Y, Tsunoda T, Takamura H, Katayama T, Kimura R, Kamino K, Hashizume Y, Takeda M: Transcriptome analysis of distinct mouse strains reveals kinesin light chain1 splicing as an amyloid-beta accumulation modifier. Proc Natl Acad Sci U S A 2014, 111:2638-2643

47. Inomata H, Nakamura $Y$, Hayakawa A, Takata H, Suzuki T, Miyazawa K, Kitamura N: A scaffold protein JIP-1b enhances amyloid precursor protein phosphorylation by JNK and its association with kinesin light chain 1. J Biol Chem 2003, 278:22946-22955

48. Kamal A, Stokin GB, Yang Z, Xia CH, Goldstein LS: Axonal transport of amyloid precursor protein is mediated by direct binding to the kinesin light chain subunit of kinesin-I. Neuron 2000, 28:449-459 